\title{
Microvascular architecture of early esophageal neoplasia
}

\author{
MAKOTO KAGA $^{1}$, HARUHIRO INOUE $^{1}$, SHIN-EI KUDO ${ }^{1}$ and SHIGEHARU HAMATANI ${ }^{2}$ \\ ${ }^{1}$ Digestive Disease Center, and ${ }^{2}$ Department of Pathology, \\ Showa University Northern Yokohama Hospital, Yokohama, Kanagawa, Japan
}

Received May 12, 2011; Accepted June 30, 2011

DOI: $10.3892 /$ or.2011.1398

\begin{abstract}
Progress in magnifying endoscopy has allowed endoscopic atypia to be evaluated on the basis of the presence or the absence of microvascular hyperplasia in a tumor. We focused our attention on intra-epithelial papillary capillary loops (IPCLs) and studied 20 cases of esophageal neoplasia (IPCL type III, 10 cases; IPCL type IV, 10 cases) and 99 vessels (IPCL type III, 24 vessels; IPCL type IV, 75 vessels). We evaluated the histopathological findings and measured vessel caliber, distance from the basement membrane, distance between blood vessels and thickness of the epithelium. According to the Vienna classification, the histological findings in the 10 patients with IPCL type III lesions were classified as category 1 (negative for neoplasia/dysplasia) in 8 patients and category 3 (non-invasive low grade neoplasia) in 2 patients. The histological findings in the 10 patients with IPCL type IV lesions were classified as category 1 in 1 patient, category 3 in 4 patients and category 4 (non-invasive high grade neoplasia) in 5 patients. The vessel caliber of IPCL type IV lesions (mean, $5.9 \pm 2.7 \mu \mathrm{m}$ ) was significantly larger than that of IPCL type III lesions (mean, $4.8 \pm 1.5 \mu \mathrm{m})(\mathrm{P}=0.013)$. The distance from the basement membrane of IPCL type IV lesions (mean, 99.9 $\pm 34.4 \mu \mathrm{m}$ ) was significantly greater than that of IPCL type III lesions (mean, 58.0 $\pm 36.2 \mu \mathrm{m})\left(\mathrm{P}=1.52562 \mathrm{E}^{-06}\right)$. The distance between blood vessels and the thickness of the epithelium did not differ significantly between IPCL type III and IPCL type IV lesions. Our results revealed that changes in vessels of IPCL type IV lesions involve two factors: increased vessel caliber and prolongation of IPCLs toward the surface. These vascular changes appear to be associated with increased atypia of blood vessels.
\end{abstract}

\section{Introduction}

In category 3 of the Vienna classification (non-invasive low grade neoplasia-low grade adenoma/dysplasia), neoplasia is

Correspondence to: Dr Makoto Kaga, Digestive Disease Center, Showa University Northern Yokohama Hospital, Chigasaki Chuo 35-1, Tsuzuki-ku, Yokohama, Kanagawa 224-8503, Japan

E-mail:kagamako.8653@gmail.com

Key words: microvascular architecture, intra-epithelial papillary capillary loops, early esophageal neoplasia present, but it is associated with a low risk of progression to invasive carcinoma. Therefore, clinicians may consider local treatment of the lesion or opt for follow-up. In category 4 (noninvasive high grade neoplasia), the risks of invasion and the development of metastases are increased. Local treatment such as endoscopic mucosal resection or local surgical treatment is therefore indicated (1). Therefore, the ability to distinguish category 3 lesions from category 4 lesions endoscopically would be beneficial. We previously noted that category 3 and category 4 lesions differ with respect to changes in blood vessels, i.e., the formation of intra-epithelial papillary capillary loops (IPCLs) (2-4).

Superficial blood vessels in the esophageal mucosa are branching vessels that extend horizontally immediately above the lamina muscularis mucosa. Intrapapillary capillaries arise from these branching vessels in epithelial papillae and form single loops referred to as IPCLs (Fig. 1). Branching vessels are observed as we approach the normal mucosa on conventional endoscopy. Using magnifying endoscopy with a scope which can magnify objects up to approximately 90 times, IPCLs appear as red dots. Recently developed narrow band imaging (NBI) systems (5) enable a more vivid depiction of IPCLs. Since IPCLs are located near the epithelium basement membrane, changes in IPCL patterns are thought to reflect alterations in the structure of the epithelial basal layer. IPCLs inside cancer lesions show abnormal changes, such as dilatation, meandering, irregular caliber, and non-uniform shapes. IPCLs are classified from type I (normal mucosa) to type V (carcinoma in situ) in accordance with the degree of atypia $(6,7)$.

The focus of our research is concerned with how to distinguish IPCL type III from IPCL type IV lesions. Approximately $95 \%$ of IPCL type III lesions correspond to category 3 (non-invasive low grade neoplasia) of the Vienna classification histologically. In contrast, approximately half of all IPCL type IV lesions are category 4 (non-invasive high grade neoplasia) or higher (8). We, therefore, routinely follow up IPCL type III lesions and endoscopically resect IPCL type IV lesions. Upon endoscopic examination, IPCL type III lesions appear as unstained areas on iodine staining or as brownish areas on NBI; changes in IPCLs in these areas are nearly identical to those in the background mucosa (Fig. 2). In contrast, IPCL type IV lesions appear more distinctly as non-iodine-stained areas on conventional endoscopy or brownish areas on NBI, and IPCLs in the lesions show mild to moderate changes (Fig. 3). Although these changes are 


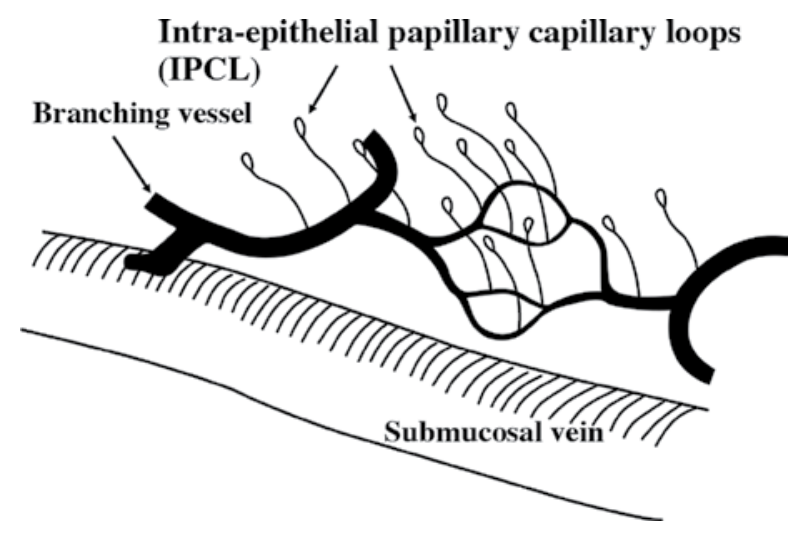

Figure 1. Schematic drawing of superficial blood vessels in the squamous epithelium on magnifying endoscopy. Branching vessels extend horizontally immediately above the lamina muscularis mucosae. Intra-epithelial papillary capillary loops (IPCLs) arise from branching vessels perpendicularly. IPCLs are located in papillae of the squamous epithelium.

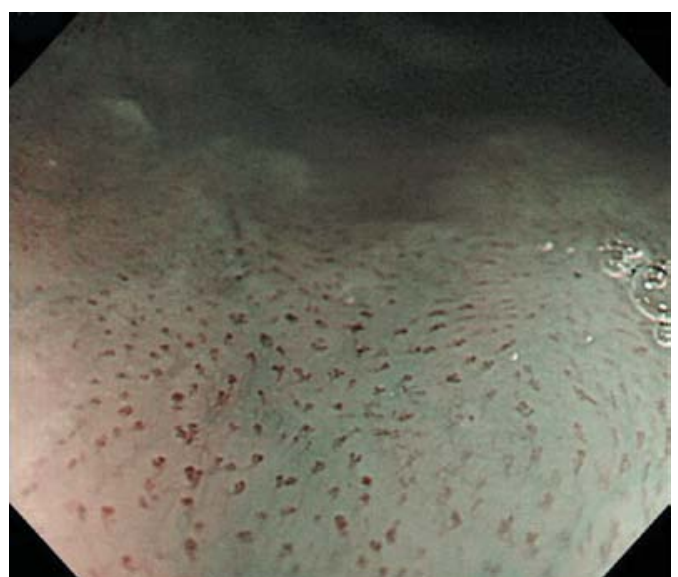

Figure 2. NBI imaging of a lesion of IPCL type III. The IPCL type III lesion appears as a non-iodine-stained area or as a brownish area on NBI imaging, and the IPCLs in the lesion show no appreciable changes as compared with the background tissue.

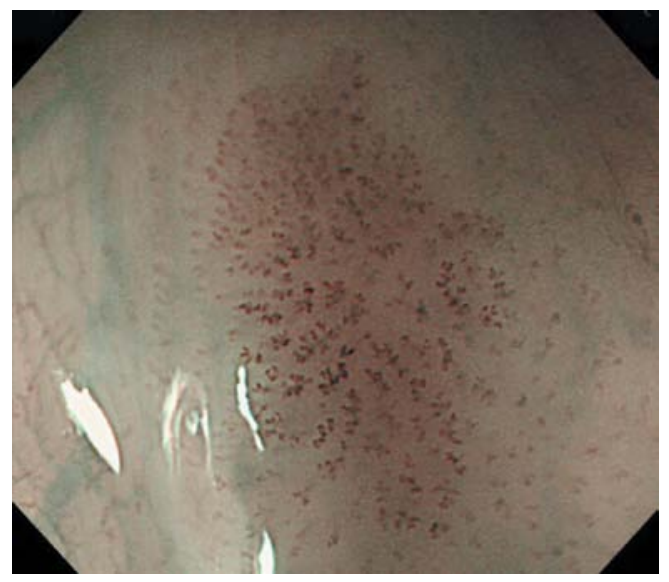

Figure 3. NBI imaging of a lesion of IPCL type IV. The IPCL type IV lesion is recognized clearly as a non-iodine-stained area or a brownish area on NBI imaging, and the IPCLs in the lesion are altered slightly or moderately.

known to occur, to our knowledge no study has compared the underlying histopathological changes between IPCL type III and IPCL type IV lesions. Thus, the aim of the present

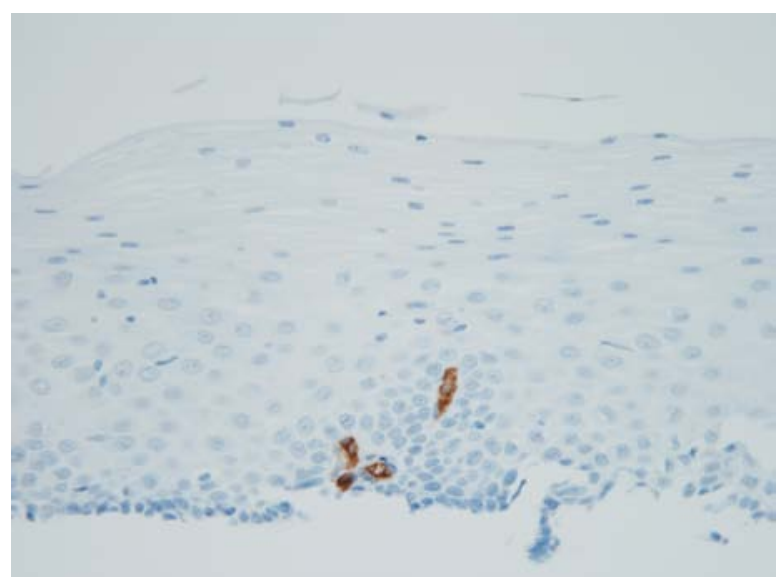

Figure 4. Histological view of the section shown in Fig. 2 (CD34, x400).

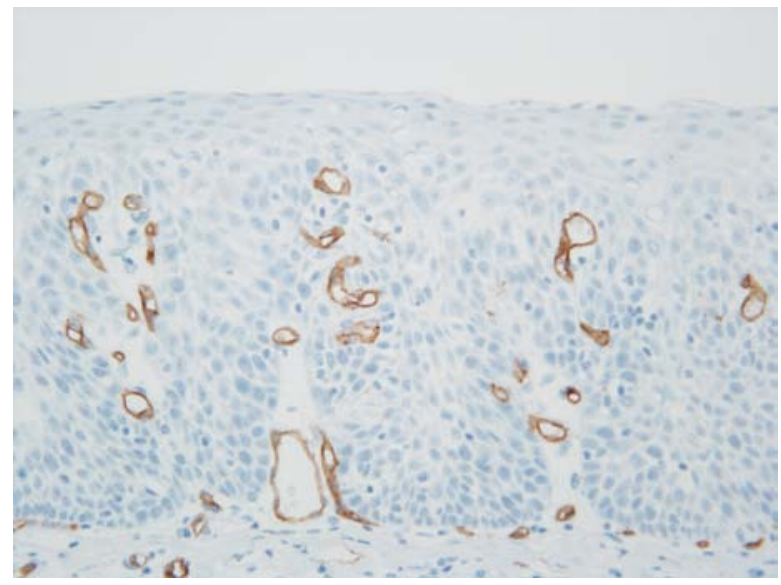

Figure 5. Histological view of the section shown in Fig. 3 (CD34, x400).

study was to examine whether IPCL type III and IPCL type IV lesions can be distinguished endoscopically as well as histologically. 


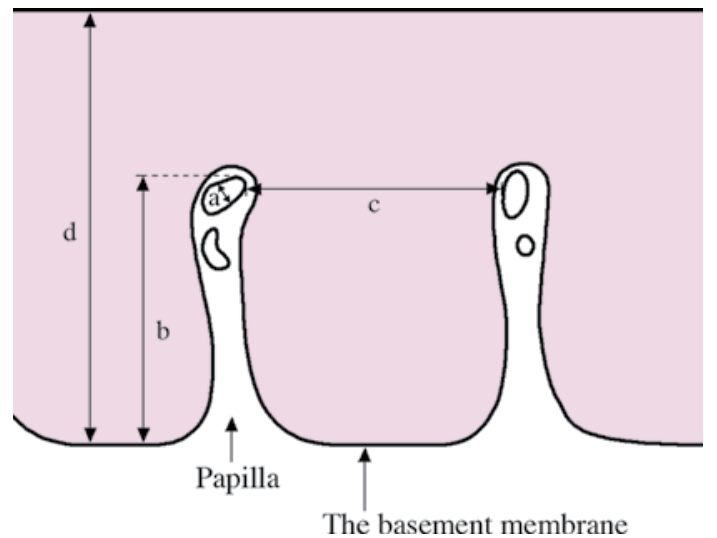

Figure 6. Schematic drawing of the papilla and blood vessels. (a) Vessel caliber; (b) distance from the basement membrane; (c) distance between blood vessels; and (d) thickness of the epithelium.

\section{Materials and methods}

From January 2005 through December 2009, 126 cases of esophageal neoplasia were diagnosed as IPCL type III or IPCL type IV lesions and patients underwent biopsy or endoscopic excision at Showa University Northern Yokohama Hospital. An EVIS 240 video endoscope system (Olympus Medical Systems) was used from January 2005 through March 2006, and a Spectrum NBI system (Olympus Medical Systems) was used from April 2006 through December 2009. An ultra-high magnifying endoscope (GIF-H260Z, GIF-H240, Olympus Medical Systems Co., Ltd., Tokyo, Japan), which can optically magnify objects up to $\sim$ x90, was employed in our study. A soft black plastic hood (MB-46, Olympus Co.) was attached to the endoscope to obtain a clearer view upon magnification.

We examined 20 cases (IPCL type III, 10 cases; IPCL type IV, 10 cases) and 99 vessels (IPCL type III, 24 vessels; IPCL type IV, 75 vessels) in which IPCLs were clearly visible on NBI and evaluated the vessels histologically. The 10 patients with IPCL type III lesions included 8 men and 2 women with a median age of 66.8 years (range, 59-74). Nine patients underwent a biopsy, and 1 underwent endoscopic treatment. The 10 patients with IPCL type IV lesions consisted of 7 men and 3 women with a median age of 69.0 years (range, 62-77). One patient underwent a biopsy, and 9 received endoscopic treatment. All patients provided informed consent for the use of their tumor samples for research purposes.

Parts of the lesions that showed IPCLs on magnifying endoscopy were split vertically and sliced at $2-\mathrm{mm}$ intervals. The biopsy specimens in particular were extended with the use of an acupuncture needle (KN-119, Kanaken, Yokohama, Japan) and cut perpendicularly. The specimens were embedded in paraffin and thinly sliced into $3-\mu \mathrm{m}$ sections. The sections were stained with hematoxylin and eosin and immunohistochemical staining for CD-34 and D2-40 was carried out. Slides were examined with a light microscope and projected on a color monitor (Olympus AX-80; Olympus Co). Slides stained with hematoxylin and eosin were used as a reference, and lumens that were positive for CD-34 immunohistochemical staining and negative for D2-40 immunohistochemical staining were identified as blood vessels (Figs. 4 and 5). Sites with the highest vascular density that were located within $150 \mu \mathrm{m}$ from the basement membrane were measured at a magnification of x200 or x400. Large vessels with a lumen diameter of $50 \mu \mathrm{m}$ or greater were excluded from the count.

The vessel caliber, distance from the basement membrane, distance between blood vessels, and the thickness of the epithelium were measured (Fig. 6). Vessel caliber was defined as the shortest diameter between the medial surfaces of endothelial cells. The distance from the basement membrane was defined as the distance from the basement membrane to the upper margin of the vessel. The distance between blood vessels was measured within 50 to $70 \mu \mathrm{m}$ from straight lines dividing the epithelium into three layers of equal thickness. When only one or no blood vessel was present near the line, the measurement of the distance between blood vessels was considered not possible. The thickness of the epithelium was also measured in each case. Each distance was measured using Sensive Measure 1.5 (Visual System Division, Mitani Corp., Tokyo, Japan).

Statistical analysis. The t-test was used to evaluate differences in vessel caliber and the distance from the basement membrane. The Mann-Whitney U test was used to evaluate the distance between blood vessels and the thickness of the epithelium. All P-values presented are two-sided. P-values of $<0.05$ were considered to indicate statistical significance. All analyses were performed using Statmate III, version 3.16 (ATMS, Tokyo, Japan).

\section{Results}

Histopathological findings. According to the Vienna classification, the histological findings of the 10 patients with IPCL type III lesions were classified as category 1 (negative for neoplasia/dysplasia) in 8 patients and category 3 (non-invasive low grade neoplasia) in 2. The histological findings of the 10 patients with IPCL type IV lesions were classified as category 1 (negative for neoplasia/dysplasia) in 1 patient, category 3 (non-invasive low grade neoplasia) in 4 , and category 4 (noninvasive high grade neoplasia) in 5.

Histomorphometric analysis. The vessel caliber of IPCL type IV (mean, $5.9 \pm 2.7 \mu \mathrm{m}$ ) was significantly larger than that of IPCL type III (mean, $4.8 \pm 1.5 \mu \mathrm{m})(\mathrm{P}=0.013)$. The distance from the basement membrane of IPCL type IV (mean, $99.9 \pm 34.4 \mu \mathrm{m}$ ) was significantly greater than that of IPCL type III (mean, 58.0 $\pm 36.2 \mu \mathrm{m})\left(\mathrm{P}=1.52562 \mathrm{E}^{-06}\right)$. The distance between blood vessels and the thickness of the epithelium did not differ significantly between IPCL type III and IPCL type IV lesions (Table I).

\section{Discussion}

The relationship between angiogenesis of esophageal squamous cell carcinoma and clinicopathological features has been demonstrated in several studies (9-13). In early esophageal carcinoma, Kuwano et al reported that the vessel counts of carcinoma in situ or microinvasive cancerous lesions differed significantly from that of adjacent normal tissue (14). Aharinejad et al prepared vascular corrosion casts by using scanning electron microscopy to study the three-dimensional 
Table I. Comparison of histomorphometric variables between IPCL type III and IPCL type IV lesions.

\begin{tabular}{|c|c|c|c|c|c|}
\hline \multirow[b]{2}{*}{ Variable } & \multicolumn{2}{|r|}{ IPCL type III } & \multicolumn{2}{|r|}{ IPCL type IV } & \multirow[b]{2}{*}{ Significance } \\
\hline & $\mathrm{n}$ & mean $\pm \mathrm{SD}$ (range) in $\mu \mathrm{m}$ & $\mathrm{n}$ & mean $\pm \mathrm{SD}$ (range) in $\mu \mathrm{m}$ & \\
\hline Vessel caliber & 24 & $4.8 \pm 1.5(2.5-8.7)$ & 75 & $5.9 \pm 2.7(2.2-12.3)$ & $\mathrm{p}=0.013$ \\
\hline Distance from the basement membrane & 24 & $58.0 \pm 36.2(18-141.3)$ & 75 & $99.9 \pm 34.4(17-161.8)$ & $\mathrm{p}=1.52562 \mathrm{E}^{-06}$ \\
\hline Distance between blood vessel & 6 & $68.7 \pm 43.1(4.2-120.1)$ & 15 & $38.5 \pm 32.8(5.7-88.3)$ & N.S. \\
\hline Thickness of the epithelium & 10 & $272.0 \pm 62.2(191.2-371.8)$ & 10 & $255.1 \pm 83.9(148.1-442.9)$ & N.S. \\
\hline
\end{tabular}

SD, standard deviation; N.S., not significant. Only significant P-values are shown.

structure of the esophageal microvascular architecture (15). They described IPCLs as 'capillary loops protruding towards the lumen' in the esophagus of the guinea pig. However, they reported that capillary loops of the mucosa were present only in guinea pigs and rats. Inoue et al examined the esophageal vascular architecture by ultra-high magnification endoscopy and for the first time successfully observed IPCLs $(3,4)$. They also reported that superficial esophageal carcinomas show characteristic changes in IPCLs according to the depth of invasion. Kumagai et al examined surgically resected specimens of superficial esophageal cancer by stereoscopic microscopy, using the Microfil injection technique (2). Furthermore, they histomorphometrically measured the caliber of superficial blood vessels of esophageal tumors and found that vessel caliber gradually increased in parallel to the depth of invasion (16).

Category 3 lesions (non-invasive low grade neoplasia) according to the Vienna classification can be followed up, whereas treatment is indicated for category 4 lesions (non-invasive high grade neoplasia) (1). Using endoscopic examination, vascular changes are the only factor that can be used to distinguish category 3 from category 4 lesions. We therefore focused on changes in IPCLs, particularly differences between IPCL type III and IPCL type IV lesions. As stated above, approximately $95 \%$ of IPCL type III lesions correspond to category 3 of the Vienna classification, and about half of all IPCL type IV lesions correspond to category 4 (8). The aim of this study was to examine whether IPCL type III and IPCL type IV lesions can be distinguished histologically. We found that IPCL type III and IPCL type IV lesions did not differ significantly with respect to the distance between blood vessels or the thickness of the epithelium. In contrast, vessel caliber and the distance from the basement membrane were significantly greater in IPCL type IV lesions than in IPCL type III lesions. The distance between papillae containing IPCLs was similar in IPCL type III lesions and IPCL type IV lesions, but IPCL type IV lesions had greater vessel calibers and more vessels extending upwards toward the surface than did IPCL type III lesions. In short, our results showed that changes in vessels of IPCL type IV lesions involve two factors: increased vessel caliber and prolongation of IPCLs toward the surface. The P-values suggest that the change in the prolongation of IPCLs had a stronger impact than increased vessel caliber.

Of the 10 IPCL type III lesions studied, 8 were category 1 according to the Vienna classification (negative for neoplasia/ dysplasia) and 2 were category 3 (non-invasive low grade neoplasia). Among the 10 IPCL type IV lesions, 1 was category 1,3 were category 3 , and 5 were category 4 (non-invasive high grade neoplasia). Therefore, the differences between IPCL type III and IPCL type IV lesions appear to reflect initial changes in IPCLs occurring during the transition from normal mucosa to non-invasive high grade neoplasia. Such changes in IPCLs may be detected on magnifying endoscopy using an NBI system.

In conclusion, we studied the differences between IPCL type III and IPCL type IV lesions that may be used to determine a policy for endoscopic treatment, and we compared histopathological changes associated with these two types of lesions. Our results showed that changes in vessels of IPCL type IV lesions involve two factors: increased vessel caliber and prolongation of IPCL toward the surface. These vascular changes appear to be associated with increased atypia of blood vessels.

\section{Acknowledgements}

We are indebted to Professor Chiaki Nishimura from the Department of Medical Informatics, Toho University School of Medicine for statistical assistance and to Mr. Peter Star from Medical Network K.K. for assistance with the manuscript.

\section{References}

1. Schlemper RJ, Riddell RH, Kato Y, et al: The Vienna classification of gastrointestinal epithelial neoplasia. Gut 47: 251-255, 2000.

2. Kumagai Y, Toi M and Inoue H: Dynamism of tumour vasculature in the early phase of cancer progression: outcome from oesophageal cancer research. Lancet Oncol 3: 604-610, 2002.

3. Inoue H, Honda T, Nagai K, Kawano T, Yoshino K, Takeshita K and Endo M: Ultra-high magnification endoscopic observation of carcinoma in situ of the esophagus. Dig Endosc 9: 16-18, 1997.

4. Inoue $\mathrm{H}$, Honda $\mathrm{T}$, Yoshida $\mathrm{T}$, et al: Ultra-high magnification endoscopy of the normal mucosa. Dig Endosc 8: 134-138, 1996.

5. Gono K, Yamazaki K, Douguchi N, et al: Endoscopic observation of tissue by Narrowband illumination. Opt Rev 10: 211-215, 2003.

6. Inoue H, Kaga M, Sato Y, Sugaya S and Kudo S: Magnifying endoscopic diagnosis of tissue atypia and cancer invasion depth in the area of pharyngo-esophageal squamous epithelium by NBI enhanced magnification image: IPCL pattern classification. In: Comprehensive Atlas of High Resolution Endoscopy and Narrowband Imaging. Cohen J (ed). Blackwell Publishing Limited, Oxford, pp49-66, 2007.

7. Inoue H: Magnifying endoscopy in the esophagus and stomach. Dig Endosc 13: S40-S41, 2001. 
8. Inoue H: The endoscopic diagnosis and treatment of the esophageal carcinoma. Igaku-shoin, Tokyo, pp14-25, 2009 (In Japanese).

9. Tanigawa $\mathrm{N}$, Matunura M, Amaya $\mathrm{H}$, et al: Tumor vascularity correlates with the prognosis of patients with esophageal squamous cell carcinoma. Cancer 79: 220-225, 1997.

10. Torres C, Wang H, Turner J, et al: Prognostic significance and effect of chemoradiotherapy on microvessel density (angiogenesis) in esophageal Barrett's esophagus-associated adenocarcinoma and squamous cell carcinoma. Hum Pathol 30: 753-758, 1999.

11. Millkan KW, Mall JW, Myers JA, Hollinger EF, Doolas A and Saclarides TJ: Do angiogenesis and growth factor expression predict prognosis of esophageal cancer? Am Surg 66: 401-406, 2000.

12. Takebayashi Y, Natugoe S, Baba M, et al: Angiogenesis in esophageal squamous cell carcinoma. Oncol Rep 5: 401-404, 1998.
13. Igarashi M, Dhar DK, Kubota H, Yamamoto A, EL-Assal O and Nagasue N: The prognostic significance of microvessel density and thymidine phosphorylase expression in squamous cell carcinoma of the esophagus. Cancer 82: 1225-1232, 1998.

14. Kuwano H, Sonoda K, Yasuda M, Sumiyoshi K, Nozoe T and Sugimachi K: Tumor invasion and angiogenesis in early esophageal squamous cell carcinoma. J Surg Oncol 65: 188-193, 1997.

15. Aharinejad S, Lametschwandtner A, Franz P and Firbas W: The vascularization of the digestive tract studied by scanning electron microscopy with special emphasis on the teeth, esophagus, stomach, small and large intestine, pancreas, and liver. Scanning Microsc 5: 811-849, 1991.

16. Kumagai $Y$, Inoue $H$, Nagai K, Kawano H and Iwai T: Magnifying endoscopy, stereoscopic microscopy, and the microvascular architecture of superficial esophageal carcinoma. Endoscopy 34: 369-375, 2002 